\title{
Editorial
}

\section{CDDis celebrates 1000 publications of essential research}

\author{
EH Baehrecke ${ }^{\star, 1}$ \\ Cell Death and Disease (2014) 5, e1042; doi:10.1038/cddis.2014.19; published online 30 January 2014
}

Cell Death and Disease (CDDis) has reached a significant milestone-1000 publications. This is a remarkable accomplishment to achieve in less than 5 years from the start of this journal. These papers emanate from laboratories in many countries, illustrating the strong international presence of CDDis. Importantly, the publication of this number of papers in a new journal with a 5-year impact factor that is greater than 6 demonstrates that $C D D$ is fills an important niche in the communication of science.

As biologists, we exist at a remarkable stage in the history of science. Technologies, including robust and inexpensive genome sequencing, numerous screening technologies, novel molecular imaging approaches, the ability to work across multiple model systems, and others, have positioned us to make discoveries at a rapid rate. Now the goal of studying a fundamental cellular process with the hope of improving our society is in the hands of relatively small research teams. A number of success stories exist, including how studies of clams, yeast and other simple organisms facilitated our understanding of the cell cycle and spawned the concept of chemical modulators of cell division as a therapeutic strategy. Similarly, the discovery of the apoptosis pathway in nematodes provided motivation to screen for therapies based on the modulation of cell death. However, a daunting gap remains between pioneering fundamental discoveries and the development of therapeutic strategies that can be translated to influence practice in clinical settings.

A key component of bridging discovery-based research to the clinic is communication of results, and CDDis is uniquely positioned to contribute to this goal. CDDis is committed to the rapid online and open access communication of top-quality fundamental discoveries with the potential to have future impact. Of course, it is difficult to predict where the next major discovery with potential translational impact exists, so as scientists, reviewers and editors, the emphasis has to be on the quality of science; quality can be evaluated and will last such that a discovery has future value. This said, we must avoid excessive revisions once appropriate quality and quantity of discovery are in place, as discovery never ends and most research leads to more questions than answers. Therefore, as editors we must help authors navigate complex reviewer comments, and facilitate publication.
Many issues challenge our community during this period of rapid discovery and communication. Importantly, we must worry about issues of data quality and originality. These are serious issues, and CDDis has established new guidelines and procedures to deal with such concerns. In addition, we must do more to improve our communication with other scientists, as well as non-specialists that help society understand the value of discovery-based research. Our society is increasingly dependent on social media outlets, such as Twitter and Facebook, but we must consider additional options. CDDis has a clear role in the communication of results to experts, and we need to consider creative approaches to communicate with our society, such as venues that merge either science with fashion (http://www.fashiondescience.com/) or science with art (http://ilovememphisblog.com/ 2013/09/st-jude-scientists-local-artists-art-of-science-2013/).

CDDis communicates the results of discovery-based research. Traditionally, this has been called basic research, and we should reconsider the use of this term. The term basic research implies both elementary and simplistic concepts, and discovery-based research is far from being simple. I prefer the terms fundamental and essential research, as these words more accurately reflect the foundation that is laid by discovery-based research. Perhaps fundamental is the best term, as my hope would be to reserve the term essential research to reflect the science that bridges fundamental and clinical sciences. Pharmacologists and the pharmaceutical industry have traditionally resided at the interface between fundamental scientists and clinical application. In this framework, CDDis publishes both fundamental and essential science, and facilitates the essential goal of the application of fundamental discoveries.

Several goals should be considered as we work toward improving essential science, and I intend to involve CDDis in these goals. It is important to increase communication between fundamental and clinical scientists. A critical component of this goal is an enhanced understanding of the importance of research that is done by both of these groups so that they work together to solve important problems. Another objective is to increase the public understanding of fundamental science as the foundation of all applied science. A crucial aspect of this goal is to better document the impact of discovery. For example, scientists and their advocates should

\footnotetext{
Department of Cancer Biology, University of Massachusetts Medical School, Worcester, MA, USA

*Corresponding author: EH Baehrecke, Department of Cancer Biology, University of Massachusetts Medical School, Worcester, MA, USA. Tel: + 15088566733 ; Fax: +1 508856 1310; E-mail: Eric.Baehrecke@umassmed.edu
} 
document the economic impact of discoveries, as these data will help governments and other organizations to understand the value of investing in discovery. Although CDDis is unlikely to gather the latter information, we can help to facilitate an understanding of the value of these goals, as they will enhance both science and our society. Importantly, authors should always reference the publication of the pioneering discovery rather than the review on the subject, as this helps to promote the value of discovery-based research.

Science is at a critical junction with the potential for exponential growth in knowledge at the same time that most sources of scientific funding are decreasing. This is an alarming issue, and we should not isolate ourselves to only focus on research and publications-we must be strong advocates for the pursuit of education and knowledge.
Therefore, CDDis and our authors must work together to improve the future of science. We must facilitate the education of our youth, including future scientists, and increase the awareness of other scientists and our society about the importance of discovery-based research. I envision CDDis playing an important role in this process.

\section{Conflict of Interest}

The authors declare no conflict of interest.

Cell Death and Disease is an open-access journal published by Nature Publishing Group. This work is licensed under a Creative Commons Attribution-NonCommercialNoDerivs 3.0 Unported License. To view a copy of this license, visit http://creativecommons.org/licenses/by-nc-nd/3.0/ 